\title{
Urate crystal deposition in hyperuricemic children: a dual energy computed tomography study
}

\author{
Qianqian Ying ${ }^{1}$, Jiapei Wang ${ }^{2}$, Yunyan $\mathrm{Li}^{2}$, Nan Sun ${ }^{3}$, Yazhen $\mathrm{Di}^{2}$, Mengjiao Shen ${ }^{2}$, Shiwei Fu
}

\begin{abstract}
${ }^{1}$ Medicine School of Ningbo University, Ningbo, Zhejiang, China
2Department of Pediatric Rheumatoid Immunity, Ningbo Women and Children's Hospital, Ningbo, Zhejiang, China

${ }^{3}$ Department of Pediatric Immunity, Tianjin Children's Hospital, Tianjin, China
\end{abstract}

Submitted: 5 February 2018

Accepted: 9 April 2018

Arch Med Sci 2021; 17 (1): 100-105

DOI: https://doi.org/10.5114/aoms/89835

Copyright $\odot 2020$ Termedia \& Banach

\section{Abstract}

Introduction: The incidence of hyperuricemia (HUA) at younger ages is increasing along the coastal regions of China. This study aimed to compare the frequency of dual energy CT (DECT) urate crystal deposition between symptomatic hyperuricemic children and asymptomatic hyperuricemic children.

Material and methods: Fifty-six hyperuricemic children were divided into a Joint Group $(n=33)$ and an Asymptomatic Group $(n=23)$ according to whether they had a history of arthritis symptoms, which includes rapid onset monoarthritis with intense pain and swelling. We analyzed DECT scans of their feet from the Joint Group and the Asymptomatic Group and compared their clinical features.

Results: DECT urate deposits were observed in $28 / 33$ (84.8\%) children with symptomatic HUA and 14/23 (60.9\%) with asymptomatic HUA. We found 60 areas of urate deposition in the Joint Group; DECT urate crystal deposition was most frequently observed in the first metatarsophalangeal (MTP) joint (30.0\%), ankle joint (15.0\%), and calcaneus (13.3\%). 39 urate deposits were found in the Asymptomatic Group; DECT urate crystal deposition was most frequently observed in the calcaneus (25.6\%), the first MTP joint (17.9\%), and the first phalanx (15.4\%).

Conclusions: Urate deposition can occur in children with HUA, and these deposits occur more frequently in hyperuricemic children with a history of arthritis symptoms. Also, the urate deposition in the first MTP joint and calcaneus was more prevalent than in other joints. It is important to give more attention to hyperuricemic children.

Key words: hyperuricemia, children, dual-energy computed tomography, urate crystal, joint injury.

\section{Introduction}

Hyperuricemia (HUA) can occur as a result of urate overproduction, urate underexcretion, or a combination of both. Uric acid is the end product of purine metabolism. Monosodium urate (MSU) crystals separate out when uric acid exceeds the urate solubility threshold, which promotes acute inflammation [1]. Gout is one result of HUA, and affects approximately $4 \%$ of adults in the USA according to the National Health and Nutrition Examination Survey (NHANES) [2]. With the improvement of our living standards and significant change of diet, the incidence of HUA at younger ages is increasing along the coastal regions of China [3, 4].

\author{
Corresponding author: \\ Prof. Yazhen Di \\ Department of Pediatric \\ Rheumatoid Immunity \\ Ningbo Women and \\ Children's Hospital \\ 339 Liuting St \\ Ningbo, Zhejiang 315000 \\ China \\ E-mail: dyz028sci@163.com
}


Meanwhile our previous research found that MSU crystal deposition can be observed in hyperuricemic children [5]. It suggests that some patients with HUA have developed joint disease, and it may significantly affect their quality of life. However, there is little research about MSU crystals in hyperuricemic children. The present research used dual-energy CT (DECT) to analyze the content of MSU crystals, and the data are used to compare the frequency of DECT urate crystal deposition in children with HUA.

\section{Material and methods}

Fifty-six children, including 42 boys and 14 girls aged from 5 to $17(12.13 \pm 2.05)$ years, with HUA were prospectively recruited between August 2015 to February 2018 from the Department of Pediatric Rheumatoid Immunity (inpatient and outpatient), Ningbo, China. Clinical features, laboratory data and DECT scans of both feet were obtained from participants with HUA, defined as serum urate level above the normal range for age and sex. The pediatric reference ranges were used as follows: babies aged 1-12 months 80-500 $\mathrm{mol} / \mathrm{l}$, children aged $1-10$ years $120-320 \mu \mathrm{mol} / \mathrm{l}$, boys aged 11-15 years $160-470 \mu \mathrm{mol} / \mathrm{l}$ for, and girls aged 11-15 years $140-350 \mu \mathrm{mol} / \mathrm{l}$. For those aged above 15 years the normal adult range was used $[6,7]$. We excluded participants who had secondary HUA such as renal insufficiency, medicine, hematologic diseases, hyperthyroidism, autoimmune disease, or cancer after radiotherapy or chemotherapy. Patients' serum urate was 363-757 $\mu \mathrm{mol} / \mathrm{I}$ when they underwent the foot DECT. Children with HUA were divided into two groups. Joint Group: 1) HUA; 2) A history of arthritis symptoms including sudden rapid onset monoarthritis with intense pain and swelling. Inquiring about the medical history, body examination and other assistant examination were used to exclude trauma, fracture, growing pain, tumor and leukemia, especially juvenile idiopathic arthritis. Asymptomatic Group: 1) HUA; 2) Without a history of arthritis symptoms. The study was approved by the Ethical Committee of the Ningbo Women and Children's Hospital and informed consent was obtained from the parents.

DECT scans of the feet were performed on a dual X-ray tube 128 detector row scanner (Somatom Definition, Siemens, Munich, Germany). The image reconstructions were done using proprietary software (Gout, Syngo CT Workplace, Siemens Medical Systems; Siemens, Munich, Germany). After cleaning their feet, all children had DECT scans of both feet. The scanning parameters were used as follows: tube A, $140 \mathrm{kV} / 55 \mathrm{~mA}$; tube $\mathrm{B}$, $80 \mathrm{kV} / 243 \mathrm{Ma}$, collimation $0.6 \mathrm{~mm}$ reconstructed to $0.75 \mathrm{~mm}$ thick slices. The setting of image reconstructions was used as follows: $80 \mathrm{kV}$ X-ray: soft tissue $50 \mathrm{HU}$, ratio 1.25, minimum $150 \mathrm{HU}$, range 5 , air distance 5 , the $140 \mathrm{kV}$, X-ray: soft tissue $50 \mathrm{HU}$, minimum $500 \mathrm{HU}$, bone distance 5. There were two readers (one radiologist and one rheumatologist, both experienced in DECT assessment), blinded to all clinical variables, including duration and serum urate results. They independently evaluated the DECT scans for the presence and sites of urate deposition.

\section{Statistical analysis}

Statistical analysis was performed using SPSS 18.0. Means with SD, median (range: max.-min.), percentages were used to describe the clinical characteristics of children. Differences between groups were analyzed by the $\chi^{2}$ test and $t$ test, with $p<0.05$ for the difference indicating statistical significance.

\section{Results}

\section{Clinical characteristics of children with HUA}

Table I shows the 56 children's clinical features. There were no significant difference in age, gender, or body mass index (BMI) between the two groups. Mean BMI of all children was $23.12 \mathrm{~kg} / \mathrm{m}^{2}$. There were 12 obese children in the Joint Group, and 9 in the Asymptomatic Group according to age- and sex-specific BMI cutoff points for overweight and obese in Chinese children established by the Group of China Obesity Force [8]. Twenty-five $(75.8 \%)$ patients in the Joint Group and 19 (82.6\%) patients in the Asymptomatic Group had a serum urate level above $420 \mu \mathrm{mol} / \mathrm{l}$. Mean disease duration in the Joint Group was 13.55 weeks. Four children in the Joint Group had a family history of gout or HUA, and two were sisters. Nine children in the Joint Group and 4 in the Asymptomatic Group were on urate-lowering therapy by benzbromarone and colchicine.

\section{DECT MSU deposition in children with HUA}

In the Joint Group, DECT MSU deposits were found in 28 (84.8\%) children, and 14 (60.9\%) children in the Asymptomatic Group (Table I). In total, 99 areas of urate deposition were observed in all participants. We found 60 areas of urate deposition in the Joint Group; DECT urate crystal deposition was most frequently observed in the first metatarsophalangeal (MTP) joint (30.0\%), ankle joint (15.0\%), and calcaneus (13.3\%). Ninety-nine areas of urate deposition were found in the Asymptomatic Group; DECT urate crystal deposition was most frequently observed in the calcaneus (25.6\%), the first MTP joint (17.9\%), and the first phalanx (15.4\%) (Table II, Figure 1). 
Qianqian Ying, Jiapei Wang, Yunyan Li, Nan Sun, Yazhen Di, Mengjiao Shen, Shiwei Fu

Table I. Clinical features and DECT MSU deposition rate of participants

\begin{tabular}{|lccc|}
\hline Parameter & Joint group, $n=33$ & Asymptomatic group, $n=23$ & $P$-value \\
\hline Age [years] & $12.18 \pm 1.81 ; 13(8)$ & $12.0 \pm 2.40 ; 12(12)$ & $t=0.246, p=0.807$ \\
\hline $\begin{array}{l}\text { Gender, } n(\%): \\
\text { Boy }\end{array}$ & $25(75.8)$ & $17(73.9)$ & $\chi^{2}=0.025, p=0.875$ \\
\hline Girl & $8(24.2)$ & $6(39.1)$ & \\
\hline BMI $\left[\mathrm{kg} / \mathrm{m}^{2}\right]$ & $22.80 \pm 5.47 ; 22.23(20.77)$ & $23.58 \pm 4.73 ; 23.08(18.19)$ & \\
\hline Serum urate $[\mu \mathrm{mol} / \mathrm{l}]$ & $465.61 \pm 84.26 ; 448(388)$ & $466.52 \pm 59.66 ; 472(24)$ & \\
\hline UA $\geq 420 \mu \mathrm{mol} / \mathrm{l}, n(\%)$ & $25(75.8)$ & $19(82.6)$ & \\
\hline Positive rate $n(\%):$ & & $14(60.9)$ & \\
\hline \begin{tabular}{l} 
Positive \\
\hline Negative
\end{tabular} & $28(84.8)$ & $9(39.1)$ & \\
\hline HUA duration $[$ weeks] & $13.55 \pm 22.51$ & $\mathrm{NA}$ & \\
\hline Family history, $n(\%)$ & $4(14.8 \%)$ & $1(4.3 \%)$ & \\
\hline
\end{tabular}

Unless specified, data are presented as mean $\pm S D$; median (range: max.-min.), BMI - body mass index.

Table II. Sites of urate deposition in HUA children

\begin{tabular}{|c|c|c|c|c|c|c|c|c|}
\hline Group & Data & $\begin{array}{c}1^{\text {st }} \text { MTP } \\
\text { joint }\end{array}$ & $\begin{array}{l}\text { Other MTP } \\
\text { joint }\end{array}$ & $\begin{array}{c}1^{\text {st }} \\
\text { metatarsus }\end{array}$ & $\begin{array}{c}\text { Other } \\
\text { metatarsus }\end{array}$ & $1^{\text {st }}$ phalan $x$ & $\begin{array}{c}\text { Other } \\
\text { phalanx }\end{array}$ & Calcaneus \\
\hline \multirow[t]{2}{*}{$\begin{array}{l}\text { Joint group } \\
(n=33)\end{array}$} & $\begin{array}{l}\text { MSU } \\
\text { crustal }\end{array}$ & 18 (30.0\%) & $1(1.6 \%)$ & $3(5.0 \%)$ & $4(6.7 \%)$ & $2(3.3 \%)$ & $2(3.3 \%)$ & 8 (13.3\%) \\
\hline & Patients & $13(46.4 \%)$ & $1(3.6 \%)$ & $2(7.1 \%)$ & $2(7.1 \%)$ & $2(7.1 \%)$ & $2(7.1 \%)$ & $6(21.4 \%)$ \\
\hline \multirow[t]{2}{*}{$\begin{array}{l}\text { Asymptomatic } \\
\text { group }(n=23)\end{array}$} & $\begin{array}{l}\text { MSU } \\
\text { crustal }\end{array}$ & 7 (17.9\%) & $2(5.1 \%)$ & $2(5.1 \%)$ & $1(2.6 \%)$ & 6 (15.4\%) & $2(5.1 \%)$ & $10(25.6 \%)$ \\
\hline & Patients & $5(35.7 \%)$ & 2 (14.3\%) & 2 (14.3\%) & $1(7.1 \%)$ & 5 (35.7\%) & $2(14.3 \%)$ & 7 (50.0\%) \\
\hline Group & Data & Talus & Ankle & Distal tibia & Other sites & Soft tissue & Erosion & Sum \\
\hline \multirow[t]{2}{*}{$\begin{array}{l}\text { Joint group } \\
(n=33)\end{array}$} & $\begin{array}{l}\text { MSU } \\
\text { crystal }\end{array}$ & $5(8.3 \%)$ & 9 (15.0\%) & $2(3.3 \%)$ & $4(6.7 \%)$ & $1(1.6 \%)$ & $1(1.6 \%)$ & 60 \\
\hline & Patients & 3 (10.7\%) & 7 (25.0\%) & $1(3.6 \%)$ & $3(10.7 \%)$ & $1(3.6 \%)$ & $1(3.6 \%)$ & 28 \\
\hline \multirow[t]{2}{*}{$\begin{array}{l}\text { Asymptomatic } \\
\text { group }(n=23)\end{array}$} & $\begin{array}{l}\text { MSU } \\
\text { crystal }\end{array}$ & $2(5.1 \%)$ & $2(5.1 \%)$ & $3(7.7 \%)$ & $2(5.1 \%)$ & $0(0)$ & $0(0)$ & 39 \\
\hline & Patients & $1(7.1 \%)$ & 2 (14.3\%) & 2 (14.3\%) & $2(14.3 \%)$ & $0(0)$ & $0(0)$ & 14 \\
\hline
\end{tabular}

MTP - metatarsophalangeal, MSU crystal - areas of MSU crystal deposition.

\section{Discussion}

Genetic enzyme defect, purine-rich foods and reduced renal uric acid clearance are the common risk factors for HUA [9]. We used to consider HUA as relatively rare in children when compared with adults. In our preliminary study, between June 2012 and October 2013, the incidence of HUA was 9.04\% (2099/23217) of children in Ningbo Women and Children's Hospital [4]. In Taiwan, China, the contemporary prevalence of HUA is $35.2 \%$ in children of Taiwan aborigines [10]. The results of studies seem to suggest that HUA prevalence is increasing in children. HUA is associated with kidney disease, cardiovascular disease and joint disease $[6,11,12]$. MSU crystallization can occur in children's joints with the condition progress, causing gouty arthritis and joint deformities after a series of pathophysiological changes. The present study did not reveal any significant differences in age and sex, which may be because the sample size was too limited to get accurate results. The relationship between age, sex and hyperuricemia in children needs further investigation.

In our study, we found that hyperuricemic children had significantly higher BMI than the normal range. Similar findings have been reported, indicating that $\mathrm{BMI}$ is related to uric acid concentrations $[10,13]$. The prevalence of HUA and metabolic syndrome in children with overweight and 

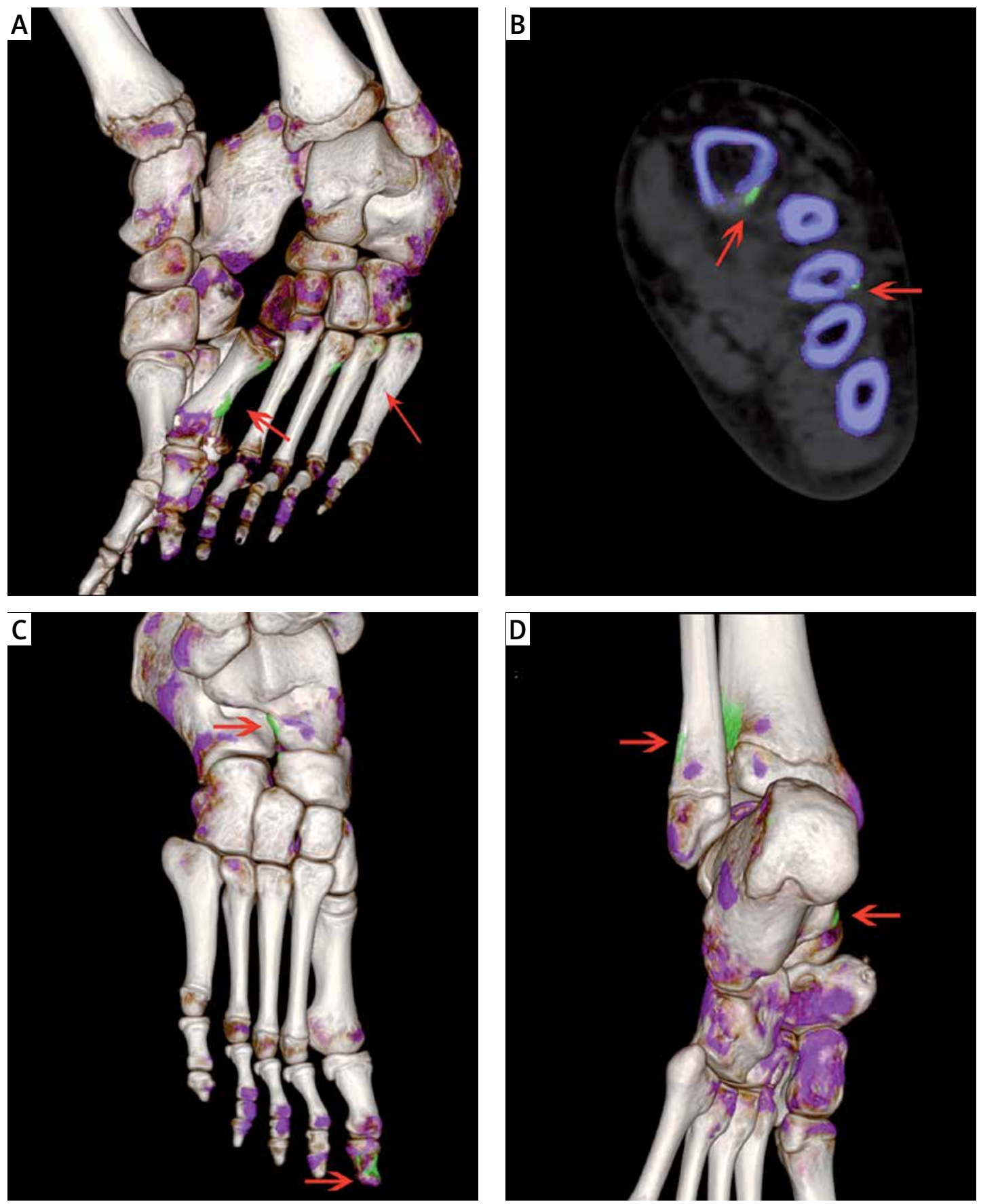

Figure 1. A, B - 10-year-old boy, HUA with left foot hallux swelling and pain. A - Three dimensional fusion, B - two dimensional coronal images show MSU crystal deposits (green) of the left metatarsals (arrows), and bone erosion. C, D - 10-year-old girl, asymptomatic HUA: C - the right foot, D - the left foot. Three dimensional fusion images show MSU crystal deposits of the right navicular bone, the right first metatarsal and lower end of left tibia and fibula, left calcaneus

obesity is high $[14,15]$. It is difficult to interpret the relationship between the uric acid concentrations and DECT MSU deposition in hyperuricemic children, because 9 children in the Joint Group and 4 in the Asymptomatic Group were on urate-lowering therapy. In addition, we observed that 5 children with HUA had a family history of gout or HUA, and two were sisters. Childhood HUA is associated with genetic factors [16-18], and it was reported that the heritability estimate for serum uric acid was $39.9 \%$ [19].

The presence of negatively birefringent needle-shaped crystals in the synovial fluid under a microscope is used as a gold standard of gout [20]. However, this is an invasive procedure and not easy to use in hyperuricemic children. DECT can identify MSU crystals, used as an advanced imaging method in the clinical treatment of gout 
and HUA in adults [21]. Moreover, DECT-detected MSU crystal deposition has been included in the 2015 American College of Rheumatology and the European League Against Rheumatism gout classification criteria [22]. Our research revealed that DECT can also identify MSU crystals in children with HUA. This is helpful to make up for the insufficiency of clinical diagnosis and to evaluate the condition of the children.

In our study, the positive rate of MSU crystals was $84.8 \%$ in the Joint Group, and one child showed bone erosion under DECT. It indicated that bone erosion can happen in hyperuricemic children like in adults with gout, which was lower than the erosion rate in adults with gout [23]. This may be related to the short time for MSU crystal deposition and thicker articular cartilage in children [24]. The positive rate of MSU crystals was $60.9 \%$ in the Asymptomatic Group. The data show that the MSU crystals appear before the joint symptoms. DECT can help us to find out whether the patients have MSU crystals, and can analyze the distribution and features of urate crystals around the feet in hyperuricemic children. Then we can implement intervention measures timely (e.g. low purine food or more water) according to the DECT results. Regular follow-up was carried out for these children to observe their uric acid levels and MSU crystal deposition, to research the relationship between the uric acid levels and joint symptoms in hyperuricemic children in the future.

Our data indicate that the most common area of deposition in symptomatic hyperuricemic children was the first metatarsophalangeal (MTP) joint (18/60), and in asymptomatic hyperuricemic children was the calcaneus (10/39), which was similar to Huppertz A's study in adults with gout [25]. This could be because the first MTP joint and calcaneus are dealing with an increase in weight load. Fewer blood vessels around and lower $\mathrm{PH}$ make this area more susceptible to MSU crystals being separated out here. Moreover, nerve endings distributed around the first MTP joint were more numerous than at the calcaneus, so it had higher sensitivity of inflammation and stimulation.

The limitation of our study was that we did not obtain patients' synovial fluid, which should be looked at under a microscope, so we cannot calculate the sensitivity and specificity of DECT for diagnosing children's gout.

In conclusion, the present study shows that MSU crystal deposition can be detected by DECT and was observed in children with HUA. In addition, it provides a comparison between symptomatic and asymptomatic children, and revealed that deposits occur more frequently in symptomatic hyperuricemic children. More importance and attention should be put on hyperuricemic children.

\section{Conflict of interest}

The authors declare no conflict of interest.

\section{References}

1. McCarty DJ, Hollander JL. Identification of urate crystals in gouty synovial fluid. Ann Intern Med 1961; 54: 452-60.

2. Zhu Y, Pandya BJ, Choi HK. Prevalence of gout and hyperuricemia in the US general population: the National Health and Nutrition Examination Survey 2007-2008. Arthritis Rheum 2011; 63: 3136-41.

3. Miao Z, Li C, Chen Y, et al. Dietary and lifestyle changes associated with high prevalence of hyperuricemia and gout in the Shandong coastal cities of Eastern China. J Rheumatol 2008; 35: 1859-64.

4. Wu L, Di YZ, Chen YL, et al. Investigation on the correlation between incidence rate of high uric acid and biochemical indicators of organ lesions among hospitalized children. Chin J Rheumatol 2014; 9: 632-6.

5. Sun N, Zhong S, Li Y, Wu L, Di Y. Urate crystals deposition in the feet of overweight juveniles and those with symptomatic hyperuricemia: a dual-energy CT study. J Pediatr Endocrinol Metab 2016; 29: 579-83.

6. Noone DG, Marks SD. Hyperuricemia is associated with hypertension, obesity, and albuminuria in children with chronic kidney disease. J Pediatr 2013; 162: 128-32.

7. Clinical Paediatric Nephrology. 3rd ed. Webb NJA, Postlethwaite RJ (eds). Oxford University Press, New York 2003; 493-509.

8. Group of China Obesity Task F. Body mass index reference norm for screening overweight and obesity in Chinese children and adolescents. Zhonghua Liu Xing Bing Xue Za Zhi 2004; 25: 97-102.

9. Agudelo CA, Wise CM. Gout: diagnosis, pathogenesis, and clinical manifestations. Curr Opin Rheumatol 2001; 13: 234-9.

10. Liu CS, Li TC, Lin CC. The epidemiology of hyperuricemia in children of Taiwan aborigines. J Rheumatol 2003; 30: 841-5.

11. Gonzalez-Senac NM, Bailen R, Torres RJ, de Miguel E, Puig JG. Metabolic syndrome in primary gout. Nucleosides Nucleotides Nucleic Acids 2014; 33: 185-91.

12. Lu CC, Wu SK, Chen HY, Chung WS, Lee MC, Yeh CJ. Clinical characteristics of and relationship between metabolic components and renal function among patients with early-onset juvenile tophaceous gout. J Rheumatol 2014; 41: 1878-83.

13. Di Y, Wang J, Chen Y, et al. Elevated interleukin 1 beta and interleukin 6 levels in the serum of children with hyperuricemia. J Clin Rheumatol 2018; 24: 65-9.

14. Ulloa-Martinez M, Burguete-Garcia Al, Murugesan S, Hoyo-Vadillo C, Cruz-Lopez M, Garcia-Mena J. Expression of candidate genes associated with obesity in peripheral white blood cells of Mexican children. Arch Med Sci 2016; 12: 968-76.

15. Civantos Modino S, Guijarro de Armas MG, Monereo Mejias S, et al. Hyperuricemia and metabolic syndrome in children with overweight and obesity. Endocrinol Nutr 2012; 59: 533-8.

16. McKinney C, Stamp LK, Dalbeth N, et al. Multiplicative interaction of functional inflammasome genetic variants in determining the risk of gout. Arthritis Res Ther 2015; 17: 288.

17. Voruganti VS, Laston S, Haack K, et al. Serum uric acid concentrations and SLC2A9 genetic variation in His- 
panic children: the Viva La Familia Study. Am J Clin Nutr 2015; 101: 725-32.

18. Chittoor G, Haack K, Mehta NR, et al. Genetic variation underlying renal uric acid excretion in Hispanic children: the Viva La Familia Study. BMC Med Genet 2017; 18: 6.

19. Wilk JB, Djousse L, Borecki I, et al. Segregation analysis of serum uric acid in the NHLBI Family Heart Study. Hum Genet 2000; 106: 355-9.

20. Pascual E, Jovani V. Synovial fluid analysis. Best Pract Res Clin Rheumatol 2005; 19: 371-86.

21. Dalbeth N, House ME, Aati O, et al. Urate crystal deposition in asymptomatic hyperuricaemia and symptomatic gout: a dual energy CT study. Ann Rheum Dis 2015; 74 908-11.

22. Neogi T, Jansen TL, Dalbeth N, et al. 2015 Gout classification criteria: an American College of Rheumatology/ European League Against Rheumatism collaborative initiative. Ann Rheum Dis 2015; 74: 1789-98.

23. Chhana A, Doyle A, Sevao A, et al. Advanced imaging assessment of gout: comparison of dual-energy CT and MRI with anatomical pathology. Ann Rheum Dis 2018; 77: 629-30.

24. Kocaoglu B, Martin J, Wolf B, Karahan M, Amendola A. The effect of irrigation solution at different temperatures on articular cartilage metabolism. Arthroscopy 2011; 27: 526-31.

25. Huppertz A, Hermann KG, Diekhoff T, Wagner M, Hamm B, Schmidt WA. Systemic staging for urate crystal deposits with dual-energy CT and ultrasound in patients with suspected gout. Rheumatol Int 2014; 34: 763-71. 